\title{
Existence Theorems for Multidimensional Lagrange Problems ${ }^{1}$
}

\author{
Lamberto Cesari ${ }^{2}$
}

\begin{abstract}
Existence theorems are proved for multidimensional Lagrange problems of the calculus of variations and optimal control. The unknowns are functions of several independent variables in a fixed bounded domain, the cost functional is a multiple integral, and the side conditions are partial differential equations, not necessarily linear, with assigned boundary conditions. Also, unilateral constraints may be prescribed both on the space and the control variables. These constraints are expressed by requiring that space and control variables take their values in certain fixed or variable sets wich are assumed to be closed but not necessarily compact.
\end{abstract}

\section{Introduction}

In the present paper, we consider multidimensional Lagrange problems of the calculus of variations of various forms, in particular, Lagrange problems where we seek the minimum of a functional of the form

$$
I[x, u]=\int_{G} f_{0}(t, x, u) d t
$$

in certain classes of pairs

$$
x(t)=\left(x^{1}, \ldots, x^{n}\right), \quad u(t)=\left(u^{1}, \ldots, u^{m}\right), \quad t=\left(t^{1}, \ldots, t^{\nu}\right) \in G \subset E_{y},
$$

satisfying (a) a system of partial differential equations each of the form

$$
\partial^{h} x^{i} / \partial^{\alpha_{1}} t^{1} \cdots \partial \partial^{\alpha_{\nu} t^{\nu}}=f_{i \alpha}(t, x, u), \quad \alpha=\left(\alpha_{1}, \ldots, \alpha_{\nu}\right), \quad h=\alpha_{1}+\cdots+\alpha_{\nu},
$$

1 Paper received March 31,1967. This research was partially supported by the Office of Scientific Research, Office of Aerospace Research, United States Air Force, Grant No. AF-AFOSR942-65.

2 Professor of Mathematics, University of Michigan, Ann Arbor, Michigan. 
(b) constraints of the form

$$
(t, x(t)) \in A \subset E_{\nu} \times E_{n}, \quad x(t) \in U(t, x(t)) \subset E_{m}
$$

(where $A$ is a given fixed set, and $U(t, x),(t, x) \in A$, is a given variable set depending on $t$ and $x$ ), and (c) a suitable system of boundary conditions concerning the values of the functions $x^{i}$ and a number of their partial derivatives on the boundary $\partial G$ of the fixed open set $G$. Here, $x$ is said to be the space variable and $u$ the control variable.

These problems are called Pontryagin problems when the sets $U(t, x)$ are all compact and contained in a fixed bounded part of the $u$-space. In the generality above, in particular when the sets $U(t, x)$ are closed and not necessarily compact subsets of $E_{m}$, these problems are called Lagrange problems with unilateral constraints on the control variable $u$. If $U(t, x)=E_{m}$ for all $t$ and $x$, then the same problems are simply called Lagrange problems (or problems without unilateral constraints in $u$ ).

In Ref. 1, we have already given existence theorems for optimal solutions for Pontryagin problems. In the present paper, we give existence theorems for optimal solutions for Lagrange problems with or without unilateral constraints. Other existence theorems will be given elsewhere. As in Ref. 1, we seek the optimal solutions in classes of pairs of functions $x, u$, with $x$ belonging to suitable Sobolev spaces and $u$ measurable. For the purpose of proving existence theorems for Lagrange problems with or without unilateral constraints, we use a technique wich is analogous to the one we used in previous papers for unidimensional $(\nu=1)$ Pontryagin and Lagrange problems (Refs. 2 and 3), and for multidimensional $(v>1)$ Pontryagin problems ( $U$ compact) (Ref. 1). The present, more difficult situation $(\nu>1$, $U$ closed but not necessarily compact) has necessitated a more subtle analysis.

\section{First Closure Theorem}

Let $G$ be a bounded open subset of the $t$-space $E_{\nu}, t=\left(t^{1}, \ldots, t^{v}\right)$; let $x=\left(x^{1}, \ldots, x^{n}\right)$ denote a vector variable in $E_{n}$ and $u=\left(u^{1}, \ldots, u^{m}\right)$ a vector variable in $E_{m}$. As usual, we shall denote by $\mathrm{cl} G$ and by bd $G=\partial G$ the closure and the boundary of $G$. We shall also denote by co $H$ the convex hull of a set $H$, and thus cl co $H$ is the closed convex hull of $H$. For every $t \in \mathrm{cl} G$, let $A(t)$ be a given nonempty subset of $E_{n}$, and let $A$ be the set of all $(t, x)$ with $t \in \operatorname{cl} G, x \in A(t)$. For every $(t, x) \in A$, let $U(t, x)$ be a subset of $E_{m}$ and let $M$ be the set of all $(t, x, u)$ with $(t, x) \in A, u \in U(t, x)$. 
The set $A$ defined above is a subset of $E_{v} \times E_{n}$, and its projection on $E_{\nu}$ is $\mathrm{cl} G$. The set $M$ defined above is a subset of $E_{v} \times E_{n} \times E_{m}$ and its projection on $E_{\nu} \times E_{m}$ is $A$.

We shall assume below that $G$ is bounded by a surface $S$ which is a regular boundary in the sense of Sobolev (see Ref. 4, Ch. 1, p. 72) and, for the sake of simplicity, we shall say that $G$ is of class $K_{l}$. Thus, $S$ can be decomposed into a finite number of manifolds $S_{1}, \ldots, S_{J}$ of dimension $n-1$ (and corresponding boundaries), each $S_{j}$ having the property that it can be mapped into a hyperplane $\pi_{j}$ by means of a transformation of coordinates $T_{j}$ defined on a part $G_{j}$ of $G$ and continuous with continuous derivatives up to ith order, $j=1, \ldots, J$.

We shall denote by $x(t)=\left(x^{1}, \ldots, x^{n}\right), u(t)=\left(u^{1}, \ldots, u^{m}\right), t \in G$, vector functions of $t$ in $G$. For every $i=1, \ldots, n$, we shall denote by $\{\alpha\}_{i}$ a given finite system of nonnegative integral indices $\alpha=\left(\alpha_{1}, \ldots, \alpha_{v}\right), 0 \leqslant|\alpha| \leqslant l_{i} \leqslant l$, with $|\alpha|=\alpha_{1}+\cdots+\alpha_{\nu}$. We shall assume that each component $x^{i}(t)$ of $x(t)$ is $L_{p_{i}}$-integrable in $G$ and possesses the generalized partial derivatives $D^{\alpha} x^{i}(t)$ of the orders $\alpha \in\{\alpha\}_{i}$, all $L_{p_{i}}$-integrable in $G$ for certain $p_{i} \geqslant 1, i=1, \ldots, n$. We shall assume that each component $u^{j}(t)$ of $u(t)$ is measurable in $G$.

Let $N$ denote the total number of indices $\alpha$ contained in the $n$ systems $\{\alpha\}_{i}, i=1, \ldots, n$, and let $f(\hat{i}, x, u)=\left(f_{i \alpha}\right)$ denote an $N$-vector function whose components are real-valued functions $f_{i \alpha}(t, x, u)$ defined on $M$. We shall consider the system of $N$ partial differential equations in $G$

$$
D^{\alpha} x^{i}=f_{i \alpha}(t, x, u), \quad \alpha \in\{\alpha\}_{i}, \quad i=1, \ldots, n,
$$

or, briefly,

$$
D x=f(t, x, u) .
$$

We are interested in pairs $x, u$ of vector functions $x(t), u(t), t \in G$, as above, satisfying the constraints

$$
(t, x(t)) \in A, \quad u(t) \in U(t, x(t)) \quad \text { a.e. in } G,
$$

and the system of partial differential equations

$$
D^{\alpha} x^{i}(t)=f_{i \alpha}(t, x(t), u(t)) \quad \text { a.e. in } G, \quad \alpha \in\{\alpha\}_{i}, \quad i=1, \ldots, n,
$$

or, briefly,

$$
D x(t)=f(t, x(t), u(t)) \quad \text { a.e. in } G .
$$

Given $\delta>0$ and a point $\left(t_{0}, x_{0}\right) \in A$, we shall denote by closed neighborhood 
$N_{\delta}\left(t_{0}, x_{0}\right)$ of radius $\delta$ of $\left(t_{0}, x_{0}\right)$ in $A$ the set of all $(t, x) \in A$ at a distance $\leqslant \delta$ from $\left(t_{0}, x_{0}\right)$. Then, by $U\left(t_{0}, x_{0} ; \delta\right)$ we shall denote the set

$$
U\left(t_{0}, x_{0} ; \delta\right)=\bigcup U(t, x),
$$

where $U$ ranges over all $(t, x) \in N_{\delta}\left(t_{0}, x_{0}\right)$. We shall say that $U(t, x)$ satisfies property $(U)$ at a point $\left(t_{0}, x_{0}\right) \in A$, provided

that is,

$$
U\left(t_{0}, x_{0}\right)=\bigcap_{\delta>0} \mathrm{cl} U\left(t_{0}, x_{0} ; \delta\right),
$$

$$
U\left(t_{0}, x_{0}\right)=\bigcap_{\delta} \mathrm{cl} \bigcup_{(t, x) \in N_{\delta}\left(t_{0}, x_{0}\right)} U(t, x)
$$

We shall say that $U(t, x)$ satisfies property $(U)$ in $A$ if $U(t, x)$ satisfies property $(U)$ at every point $\left(t_{0}, x_{0}\right) \in A$. A set $U(t, x)$ satisfying property $(U)$ is necessarily closed as the intersection of closed sets. Property $(U)$ is the socalled property of upper semicontinuity used for different purposes by G. Choquet (Ref. 5), C. Kuratowski (Ref. 6), and E. Michael (Ref. 7).

We shall consider the sets

$$
Q(t, x)=f(t, x, U(t, x))=[z \mid z=f(t, x, u), u \in U(t, x)] \subset E_{N}
$$

or other analogous sets, and assume that they are convex. We shall say that a set $Q(t, x)$ satisfies property $(Q)$ at a point $\left(t_{0}, x_{0}\right) \in A$, provided

$$
Q\left(t_{0}, x_{0}\right)=\bigcap_{\delta>0} \mathrm{clco} Q\left(t_{0}, x_{0} ; \delta\right)
$$

that is,

$$
Q\left(t_{0}, x_{0}\right)=\bigcap_{\delta} \mathrm{clco} \bigcup_{(t, x) \in N_{\delta}\left(t_{0}, x_{0}\right)} Q(t, x) .
$$

We shall say that $Q(t, x)$ satisfies property $(Q)$ in $A$ if $Q(t, x)$ satisfies property $(Q)$ at every point $\left(t_{0}, x_{0}\right) \in A$. A set $Q(t, x)$ satisfying property $(Q)$ is necessarily closed and convex as the intersection of closed and convex sets.

2.1. Closure Theorem 1. Let $G$ be a bounded open set of the $t$-space $E_{\nu}$ of some class $K_{l}, l \geqslant 1$, for every $t \in \mathrm{cl} G$ let $A(t)$ be a nonempty subset of the $x$-space $E_{n}$, and assume that the set $A$ of all $(t, x) \in E_{\nu} \times E_{n}$ with $t \in \mathrm{cl} G, x \in A(t)$ is closed. For every $(t, x) \in A$, let $U(t, x)$ be a nonempty closed subset of the $u$-space $E_{m}$ and assume that $U(t, x)$ satisfies property $(U)$ 
in $A$. Let $M$ be the set of all $(t, x, u) \in E_{v} \times E_{n} \times E_{m}$ with $(t, x) \in A$, $u \in U(t, x)$. For every $i=1, \ldots, n$, let $\left\{\alpha_{i}\right\}$ be a finite system of nonnegative integral indices $\alpha=\left(\alpha_{1}, \ldots, \alpha_{\nu}\right), 1 \leqslant|\alpha| \leqslant l_{i} \leqslant l$, and let $N$ be the total number of elements $\alpha \in\{\alpha\}_{i}, i=1, \ldots, n$. Let $f(t, x, u)=\left(f_{i \alpha}, \alpha \in\{\alpha\}_{i}\right.$, $i=1, \ldots, n)$ be a continuous $N$-vector function on the set $M$, and assume that the set $Q(t, x)=f(t, x, U(t, x))$ is a convex closed subset of the $z$-space $E_{N}$ for every $(t, x) \in A$, and that $Q(t, x)$ satisfies property $(Q)$ in $A$. Let $x, u$, and $x_{k}, u_{k}, k=1,2, \ldots$, be pairs of vector functions $x(t)=\left(x^{1}, \ldots, x^{n}\right), u(t)=$ $\left(u^{1}, \ldots, u^{m}\right), x_{k}(t)=\left(x_{k}^{1}, \ldots, x_{k}{ }^{n}\right), u_{k}(t)=\left(u_{k}^{1}, \ldots, u_{k}{ }^{m}\right), t \in G, x^{i}, x_{k}{ }^{i} \in L_{\mathbf{1}}(G)$, $i=1, \ldots, n, u^{j}, u_{k}{ }^{j}$ measurable in $G, j=1, \ldots, m$. Assume that each component $x^{i}, x_{k}^{i}$ of $x, x_{k}$ possesses generalized partial derivatives $D^{\alpha} x^{i}, D^{\alpha} x_{k}{ }^{i} \in L_{1}(G)$ of the orders $\alpha \in\{\alpha\}_{i}, i=1, \ldots, n, k=1,2, \ldots$. Assume that

$$
\begin{gathered}
\left(t, x_{k}(t)\right) \in A, \quad u_{k}(t) \in U\left(t, x_{k}(t)\right) \quad \text { a.e. in } G, \\
D^{\alpha} x_{k}{ }^{i}(t)=f_{i \alpha}\left(t, x_{k}(t), u_{k}(t)\right) \quad \text { a.e. in } G, \quad \alpha \in\{\alpha\}_{i}, \quad i=1, \ldots, n,
\end{gathered}
$$

and that

$$
\begin{gathered}
x_{k}^{i}(t) \rightarrow x^{i}(t) \text { strongly in } L_{1}(G) \text { as } k \rightarrow \infty, \\
D^{\alpha} x_{k}^{i}(t) \rightarrow D^{\alpha} x^{i}(t) \text { weakly in } L_{1}(G) \text { as } k \rightarrow \infty
\end{gathered}
$$

for all $\alpha \in\{\alpha\}_{i}, i=1, \ldots, n$. Then, there is a measurable vector function $u(t)=\left(u^{1}, \ldots, u^{m}\right), t \in G$, such that

$$
\begin{gathered}
(t, x(t)) \in A, \quad u(t) \in U(t, x(t)) \quad \text { a.e. in } G, \\
D^{\alpha} x^{i}(t)=f_{i \alpha}(t, x(t), u(t)) \quad \text { a.e. in } G, \quad \alpha \in\{\alpha\}_{i}, \quad i=1, \ldots, n .
\end{gathered}
$$

The strong convergence (3) can be replaced by pointwise convergence almost everywhere in $G$. We omit the proof of this statement since Closure Theorem 1 is a particular case of Closure Theorem 2. A proof of Closure Theorem 1 can be obtained by obvious simplifications in the one of Closure Theorem 2 .

\section{Second Closure Theorem}

Let us denote by $y=\left(x^{1}, \ldots, x^{s}\right)$ the $s$-vector made up of certain components, say $x^{1}, \ldots, x^{s}, 0 \leqslant s \leqslant n$, of the $n$-vector $x=\left(x^{1}, \ldots, x^{n}\right)$, and by $z$ the complementary $(n-s)$-vector $z=\left(x^{s+1}, \ldots, x^{n}\right)$ of $x$, so that we shall write $x=(y, z)$. We shall assume that $f(t, y, u)$ depends only on the coordinates $x^{1}, \ldots, x^{s}$ of $x$. If $x(t), t \in G$, is any vector function, we shall denote by $x(t)=$ 
$(y(t), z(t))$ the corresponding decomposition of $x(t)$ in its coordinates $y(t)=\left(x^{1}, \ldots, x^{s}\right)$ and $z(t)=\left(x^{s+1}, \ldots, x^{n}\right)$. We shall denote by $A$ a closed subset of the ty-space $E_{v} \times E_{s}$, and then $\tilde{A}=A \times E_{n-s}$ will be a closed subset of the $t x$-space $E_{\nu} \times E_{n}$. We shall consider $n$-vector functions $x(t)=(y(t), z(t))$ for which the first $s$ components, or vector $y(t)=\left(x^{1}, \ldots, x^{s}\right)$, possess certain generalized partial derivatives $D^{\alpha} x^{i}(t)$ as in Closure Theorem 1 . On the remaining $n-s$ components, or vector $z(t)=\left(x^{s+1}, \ldots, x^{n}\right)$, we shall have a different set of assumptions.

We shall consider countable systems $\{I\}$ of intervals $I=[a, b] \subset G$, $a=\left(a^{1}, \ldots, a^{\nu}\right), b=\left(b^{1}, \ldots, b^{\nu}\right), a^{j}<b^{j}, j=1, \ldots, \nu$, with the property that, for any point $t \in G$, there are hypercubes $I \in\{I\}$ with $t \in I$ and diam $I$ as small as we want. For any given function $z(t), t \in G$, we shall consider the usual differences of order $\nu$ relative to the $2^{v}$ vertices of $I$, say

$$
\begin{aligned}
& \Delta z=\Delta_{I} z=z(b)-z(a) \quad \text { if } \quad v=1, \\
& \Delta z=\Delta_{I} z=z\left(b^{1}, b^{2}\right)-z\left(b^{1}, a^{2}\right)-z\left(a^{1}, b^{2}\right)+z\left(a^{1}, a^{2}\right) \quad \text { if } \quad v=2,
\end{aligned}
$$

and so on. A nonconstant function $z(t), t \in G$, is said to be singular in $G$ with respect to the system $\{I\}$ provided $\Delta_{I} \approx$ is singular, that is, provided for almost all $t_{0} \in G$ we have (meas $\left.I\right)^{-1} \Delta z \rightarrow 0$ as $\operatorname{diam} I \rightarrow 0$, with $I$ a hypercube, $I \in\{I\}, t_{0} \in I$. As we know (Ref. 8), any interval function $\varphi(I)$ of bounded variation possesses a decomposition $\varphi(I)=\Phi(I)+S(I)$, where $\Phi$ is absolutely continuous and $S$ is singular. If $\varphi(I)$ is nonnegative, then both $\Phi$ and $S$ are nonnegative.

3.1. Closure Theorem 2. Let $G$ be a bounded open set of the $t$-space $E_{v}$ of some class $K_{l}, l \geqslant 1$, let $A(t)$ be a nonempty subset of the $y$-space $E_{s}$ defined for every $t \in \mathrm{cl} G$, and assume that the set $A$ of all $(t, y) \in E_{v} \times E_{s}$ with $t \in \mathrm{cl} G, y \in A(t)$, is closed. Let $\tilde{A}$ be the closed set $\tilde{A}=A \times E_{n-s} C$ $E_{v} \times E_{n}$. Let $U(t, y)$ denote a closed subset of $E_{m}$ for every $(t, y) \in A$, and let $M$ be the set of all $(t, y, u) \in E_{v} \times E_{s} \times E_{n}$ with $(t, y) \in A, u \in U(t, y)$. For every $i=1, \ldots, n$, let $\{\alpha\}_{i}$ be a finite system of indices $\alpha=\left(\alpha_{1}, \ldots, \alpha_{\nu}\right)$, $1 \leqslant|\alpha| \leqslant l_{i} \leqslant l$, and let $\tilde{N}$ be the total number of elements $\alpha \in\{\alpha\}_{i}$, $i=1, \ldots, n$. We assume that, for every $i=s+1, \ldots, n,\{\alpha\}_{i}$ is made up of only one element $\alpha=\alpha_{0}=(1,1, \ldots, 1)$. If $N$ denotes the number of elements $\alpha \in\{\alpha\}_{i}, i=1, \ldots, s$, then $\tilde{N}=N+(n-s)$. Let $f(t, y, u)=\left(f_{i \alpha}\right), \alpha \in\{\alpha\}_{i}$, $i=1, \ldots, n$, be a continuous $\tilde{N}$-vector function on $M$, which we may write in the form $\tilde{f}=\left(f, f_{0}\right)$, where $f$ is the $N$-vector of its first $N$ components, and $f_{0}$ the $(n-s)$-vector of its remaining components. Let $\tilde{z}=\left(z^{1}, \ldots, z^{\tilde{N}}\right)=\left(z, z_{0}\right)$ with $z=\left(z^{1}, \ldots, z^{N}\right), z_{0}=\left(z^{N+1}, \ldots, z^{\tilde{N}}\right)$ and, for every $(t, y) \in A$, let $\tilde{Q}(t, y)$ 
denote the subset of the $\tau$-space $E_{\tilde{N}}$ defined by $z=f(t, y, u), z^{i} \geqslant f_{i \alpha_{0}}(t, y, u)$, $i=N+1, \ldots, \tilde{N}, u \in U(t, y)$. Let us assume that $\tilde{Q}(t, y)$ is a closed convex subset of $E_{\widetilde{N}}$ satisfying property $(Q)$ in $A$. Also, assume that $f_{i \alpha}(t, y, u) \geqslant-c_{i \alpha}$, $\alpha \in\{\alpha\}_{i}, i=1, \ldots, s$, for all $(t, y, u) \in A$ and some constants $c_{i x} \geqslant 0$. Let $x=(y, z), u, x_{k}=\left(y_{k}, z_{k}\right), u_{k}, k=1,2, \ldots$, be functions defined on $G$, all components of $x$ and $x_{k}$ of class $L_{1}(G)$ and all components of $u$ and $u_{k}$ measurable. Assume that each component $x^{k}$ and $x_{k}{ }^{i}$ of $x$ and $x_{k}$ has generalized partial derivatives $D^{\alpha} x^{i}, D^{\alpha} x_{k}^{i}$ of the orders $\alpha \in\{\alpha\}_{i}, i=1, \ldots, n$, all of class $L_{1}(G)$, and that

with

$$
\begin{aligned}
& \left(t, y_{k}(t)\right) \in A, \quad u_{k}(t) \in U\left(t, y_{k}(t)\right) \quad \text { a.e. in } G, \\
& D^{\alpha} \mathcal{X}_{k}{ }^{i}(t)=f_{i \alpha}\left(t, y_{k}(t), u_{k}(t)\right) \quad \text { a.e. in } G,
\end{aligned}
$$

$$
\alpha \in\{\alpha\}_{i}, \quad i=1, \ldots, n, \quad k=1,2, \ldots
$$

Assume that, as $k \rightarrow \infty$, we have

$$
\begin{aligned}
x_{k}^{i}(t) & \rightarrow x^{i}(t) \text { strongly in } L_{1}(G), \quad i=1, \ldots, s, \\
D^{\alpha} x_{k}^{i}(t) & \rightarrow D^{\alpha} x^{i}(t) \text { weakly in } L_{1}(G), \quad \alpha \in\{\alpha\}_{i}, \quad i=1, \ldots, s .
\end{aligned}
$$

Assume that there is a countable system of real numbers [t] everywhere dense in $E_{1}$ such that, for all points $\left\{t_{\rho}\right\}, t_{\rho} \in G$, of the form $t_{\rho}=\left(t^{1}, \ldots, t^{\nu}\right)$, $t^{j} \in[t], j=1, \ldots, \nu$, we have

$$
x_{k}^{i}(t) \rightarrow x^{i}(t) \text { at every } i \in\left\{t_{p}\right\} \text { as } k \rightarrow \infty, \quad i=s+1, \ldots, n,
$$

and for all intervals $\{I\}, I \subset G$, with vertices in $\left\{t_{\rho}\right\}$, we have

with

$$
\int_{I} D^{\alpha} x_{k}^{i}(t) d t \rightarrow \int_{I} D^{\alpha} x^{i}(t) d t \quad \text { as } \quad k \rightarrow \infty
$$

$$
\alpha=\alpha_{0} \in\{\alpha\}_{i}, \quad i=s+1, \ldots, n .
$$

Assume that there is a decomposition $x^{i}(t)=X^{i}(t)+S^{i}(t), i=s+1, \ldots, n$, of each $x^{i}, i=s+1, \ldots, n$, into two parts $X^{i}, S^{i}$, both of class $L_{1}(G), X^{i}(t)$ with generalized partial derivative $D^{\alpha_{0}} X^{i}$ of class $L_{1}(G)$ and $S^{i}(t)$ singular. Let $Z(t)$ denote the $(n-s)$-vector $Z(t)=\left(X^{s+1}, \ldots, X^{n}\right)$. Then, there is a measurable vector function $u(t)=\left(u^{1}, \ldots, u^{m}\right), t \in G$, such that, a.e. in $G$, we have

$$
\begin{aligned}
(t, y(t)) \in A, & u(t) \in U(t, y(t)), \\
D^{\alpha} x^{i}(t)=f_{i \alpha}(t, y(t), u(t)), & \alpha \in\{\alpha\}_{i}, \quad i=1, \ldots, s, \\
D^{\times} X^{i}(t)=f_{i \alpha}(t, y(t), u(t)), & \alpha=\alpha_{0} \in\{\alpha\}_{i}, \quad i=s+1, \ldots, n .
\end{aligned}
$$


Strong convergence (8) can be replaced by pointwise convergence almost everywhere in $G$.

3.2. Remark. Closure Theorem 2 reduces to Closure Theorem 1 when $s=n$, and then $A=\tilde{A}, f=\tilde{f}, x=y$.

3.3. Proof. For every $\alpha \in\{\alpha\}_{i}, i=1, \ldots, n$, let $\varphi_{i \alpha}, \varphi_{i \alpha k}$ denote the real-valued functions defined a.e. in $G$

$$
\begin{aligned}
\varphi_{i \alpha k}(t) & =D^{\alpha} \mathcal{X}_{k}{ }^{i}(t)=f_{i \alpha}\left(t, y_{k}(t), u_{k}(t)\right), \\
\varphi_{i \alpha}(t) & =D^{\alpha} x^{i}(t), \quad i=1, \ldots, s, \\
\varphi_{i \alpha}(t) & =D^{\alpha} X^{i}(t), \quad i=s+1, \ldots, n,
\end{aligned}
$$

and let $\varphi, \varphi_{k}$ denote the $\tilde{N}$-vectors

$$
\begin{aligned}
\varphi_{k}(t) & =\left(\varphi_{i \alpha k \hat{k}}(t), \alpha \in\{\alpha\}_{i}, i=1, \ldots, n\right), \quad k=1,2, \ldots, \\
\varphi(t) & =\left(\varphi_{i \alpha}(t), \alpha \in\{\alpha\}_{i}, i=1, \ldots, n\right) .
\end{aligned}
$$

By hypothesis, $A$ is a closed subset of $E_{v} \times E_{s}, U(t, y)$ is a closed subset of $E_{m}$ for every $(t, y) \in A$, and $U(t, y)$ satisfies property $(U)$ in $A$. By Ref. 1, Section $4, M$ is a closed subset of $E_{v} \times E_{s} \times E_{m}$.

For every $t_{0} \in G, t_{0}=\left(t_{0}{ }^{1}, \ldots, t_{0}{ }^{\nu}\right)$, let $\delta_{0}=\delta_{0}\left(t_{0}\right)$ denote the distance of $t_{0}$ from $\partial G$ and let $q=q_{h}=\left[t_{0}, t_{0}+h\right]$ denote any closed hypercube $q=\left[\tilde{t}^{j} \leqslant t^{j} \leqslant \tilde{t}^{j}+h, j=1, \ldots, \nu\right]$, where $\tilde{t}^{j}, \tilde{t}^{j}+h \in[t], h$ is a positive number with $0<h<\delta_{0} / \nu, \tilde{t}^{j} \leqslant t_{0}{ }^{j} \leqslant \ddot{t}^{j}+h$, so that $t_{0} \in q \subset G$; and we have denoted here, for the sake of simplicity, by $h$ also the $\nu$-vector $(h, \ldots, h)$. For almost every $t_{0} \in G$, we have, as $h \rightarrow 0^{+}$,

$$
\begin{aligned}
h^{-v} \int_{q} x^{i}(t) d t & =x^{i}\left(t_{0}\right), \quad i=1, \ldots, s, \\
h^{-v} \int_{q} D^{\alpha} x^{i}(t) d t & \rightarrow D^{\alpha} x^{i}\left(t_{0}\right), \quad a \in\{\alpha\}_{i}, \quad i=1, \ldots, s, \\
h^{-v} \int_{q} X^{i}(t) d t & \rightarrow X^{i}\left(t_{0}\right), \quad i=s+1, \ldots, n, \\
h^{-v} \Delta_{q} S^{i} & \rightarrow 0, \quad i=s+1, \ldots, n .
\end{aligned}
$$

Also, for almost all $t \in G$, we have $\left(t, y_{k}(t)\right) \in A$ for all $k=1,2, \ldots$. The convergence $x_{k}^{i} \rightarrow x^{i}$ in $L_{1}(G)$ as $k \rightarrow \infty, i=1, \ldots, s$, as stated in (8) (that is, $y_{k} \rightarrow y$ in $L_{1}(G)$ ), implies convergence in measure in $\mathrm{G}$, and hence 
there is a subsequence $\left[y_{k_{*}}(t)\right]$ which converges pointwise a.e. in $G$. Let $G_{0}$ be the set of all $t \in G$ where the relations (15) through (18) hold, where $\left(t, y_{k}(t)\right) \in A$ for all $k$, and $y_{k_{r}}(t) \rightarrow y(t)$ as $r \rightarrow \infty$. Then, $G_{0}$ is measurable with meas $G_{0}=$ meas $G$. For every $t \in G_{0}$, we have now $\left(t, y_{k_{r}}(t)\right) \in A$, where $A$ is a closed set, and as $r \rightarrow \infty$, then $(t, y(t)) \in A$ for $t \in G_{0}$, that is, $(t, y(t)) \in A$ a.e. in $G$.

Because of the convergence $y_{k}(t) \rightarrow y(t)$ in $L_{1}(G)$, and hence in measure, and consequent pointwise convergence $y_{k_{r}}(t) \rightarrow y(t)$ everywhere in $G_{0}$ with meas $G_{0}=$ meas $G$, we know that there are closed sets $C_{\lambda}, \lambda=1,2, \ldots$, with $C_{\lambda} \subset G_{0}, C_{\lambda} \subset C_{\lambda+1}$, meas $C_{\lambda}>$ meas $G_{0}-\lambda^{-1}$, such that $y(t)$ is continuous on $C_{\lambda}$ and $y_{k_{r}}(t) \rightarrow y(t)$ uniformly on $C_{\lambda}$ as $r \rightarrow \infty$ for every $\lambda=1,2, \ldots$. Since $G$ is bounded, and $C_{\lambda} \subset G_{0} \subset G$, each set $C_{\lambda}$ is compact, and hence $y(t), y_{k_{r}}(t), r=1,2, \ldots$, are continuous, uniformly continuous, and equicontinuous on each $C_{\lambda}$.

Let $\lambda$ be any fixed integer with $\lambda>(\text { meas } G)^{-1}$; hence, meas $C_{\lambda}>0$. Let $\epsilon>0$ be an arbitrary number. There is some $\delta_{0}^{\prime}=\delta_{0}^{\prime}(\epsilon, \lambda)>0$ such that $\left|t-t^{\prime}\right| \leqslant \delta_{0}^{\prime}$, with $t, t^{\prime} \in C_{\lambda}$, implies $\left|y(t)-y\left(t^{\prime}\right)\right| \leqslant \epsilon,\left|y_{k_{r}}(t)-y_{k_{r}}\left(t^{\prime}\right)\right| \leqslant \epsilon$ for every $r=1,2, \ldots$. Also, there exists some $k=k(\epsilon, \lambda)$ such that $k_{r} \geqslant k(\epsilon, \lambda), \quad t \in C_{\lambda}$, implies $\left|y_{k_{r}}(t)-y(t)\right| \leqslant \epsilon$.

Let $c>0$ be any number such that $0 \leqslant c_{i \alpha}<c$, or $0 \leqslant c_{i \alpha} / c<1$, $\alpha=\alpha_{0} \in\{\alpha\}_{i}, i=s+1, \ldots, n$.

Let $\chi_{\lambda}(t), \chi_{\lambda}{ }^{*}(t), t \in G$, be the characteristic functions of the sets $C_{\lambda}$ and $G-C_{\lambda}$, so that $\chi_{\lambda}+\chi_{\lambda}{ }^{*}=1$ everywhere in $G$. All functions $\chi_{\lambda}(t)$ and $\chi_{\lambda}(t) D^{\alpha} x^{i}(t), \alpha \in\{\alpha\}_{i}, i=1, \ldots, s$, are of class $L_{1}(G)$, and for every $t_{0} \in C_{\lambda}$ we have

$$
\chi_{\lambda}\left(t_{0}\right)=1, \quad \chi_{\lambda}^{*}(t) D^{\alpha} x^{i}\left(t_{0}\right)=0 .
$$

Then, for almost all $t_{0} \in C_{\lambda}$, we have also, as $h \rightarrow 0^{+}$,

and

$$
h^{-v} \int_{Q} \chi_{\lambda}(t) d t \rightarrow \chi_{\lambda}\left(t_{0}\right)=1
$$

with

$$
h^{-\nu} \int_{q} \chi_{\lambda}^{*}(t) D^{\alpha} x^{i}(t) d t \rightarrow \chi_{\lambda}^{*}\left(t_{0}\right) D^{\alpha} x^{i}\left(t_{0}\right)=0
$$

$$
\alpha \in\{\alpha\}_{i}, \quad i=1, \ldots, s .
$$

Let $C_{\lambda}^{\prime}$ be the subset of $C_{\lambda}$ where this occurs. Then, $C_{\lambda}$ is measurable, $C_{\lambda}^{\prime} \subset C_{\lambda} \subset G_{0} \subset G$, meas $C_{\lambda}^{\prime}=$ meas $C_{\lambda}>$ meas $G_{0}-\lambda^{-1}>0$, and, finally,

$$
\text { meas }\left(\bigcup_{\lambda} C_{\lambda}^{\prime}\right)=\text { meas } G_{0}=\operatorname{meas} G \text {. }
$$


Let $\eta>0$ be any positive number independent of $\epsilon$. Let $t_{0}$ be any point of $C_{\lambda}^{\prime}$, let $y_{0}=y\left(t_{0}\right)$, and let $u_{0}$ be any point of $U\left(t_{0}, y_{0}\right)$. Let $M_{1}>0$ be any constant such that

$$
\left|f_{i \alpha}\left(t_{0}, y_{0}, u_{0}\right)\right| \leqslant M_{1}, \quad \alpha \in\{\alpha\}_{i}, \quad i=1, \ldots, n .
$$

Let us fix $h>0$ so small that

$$
h<\epsilon / \nu, \quad h<\delta_{0} / v, \quad h<\delta_{0}^{\prime} / \nu,
$$

where

$$
\delta_{0}=\delta_{0}\left(t_{0}\right), \quad \delta_{0}^{\prime}=\delta_{0}^{\prime}(\epsilon, \lambda),
$$

and also so small that

$$
\begin{gathered}
\left|D^{\alpha} x^{i}\left(t_{0}\right)-h^{-\nu} \int_{q} D^{\alpha} x^{i}(t) d t\right|<\eta \tilde{N}^{-1}, \quad \alpha \in\{\alpha\}_{i}, \quad i=1, \ldots, s, \\
h^{-\nu} \operatorname{meas}\left(q \cap C_{\lambda}\right)=h^{-\nu} \int_{q} \chi_{\lambda}(t) d t \geqslant \max \left[2^{-1}, 1-\eta \tilde{N}^{-1} M_{1}, 1-\eta \tilde{N}^{-1} c^{-1}\right], \\
\left|h^{-\nu} \int_{q} \chi_{\lambda}^{*}(t) D^{\alpha} x^{i}(t) d t\right|<\eta \tilde{N}^{-1}, \quad \alpha \in\{\alpha\}_{i}, \quad i=1, \ldots, s, \\
\left|h^{-\nu} \Delta_{q} S^{i}\right|<\eta \tilde{N}^{-1}, \quad i=s+1, \ldots, n, \\
\left|D^{\alpha} X^{i}\left(t_{0}\right)-h^{-\nu} \int_{q} D^{\alpha} X^{i}(t) d t\right| \leqslant \eta \tilde{N}^{-1}, \quad \alpha=\alpha_{0} \in\{\alpha\}_{i}, \quad i=s+1, \ldots, n .
\end{gathered}
$$

This is possible because of expressions (15)-(20). Let $H$ and $H^{*}$ be the sets

$$
H=q \cap C_{\lambda}, \quad H^{*}=q-H=q-q \cap C_{\lambda}=q-C_{\lambda} .
$$

Then, we have

$$
\begin{aligned}
h^{-\nu} \text { meas } H & \geqslant \max \left[2^{-1}, 1-\eta \tilde{N}^{-1} M_{1}^{-1}, 1-\eta \tilde{N}^{-1} c^{-1}\right], \\
h^{-\nu} \text { meas } H^{*} & \leqslant \min \left[2^{-1}, \eta \tilde{N}^{-1} M_{1}^{-1}, \eta \tilde{N}^{-1} c^{-1}\right] .
\end{aligned}
$$

For any $k$, let $\bar{\varphi}_{i \alpha k}(t), t \in q$, be the function defined by

$$
\begin{aligned}
& \bar{\varphi}_{i \alpha k}(t)=\varphi_{i \alpha \alpha}(t)=f_{i \alpha}\left(t, y_{k}(t), u_{k}(t)\right) \quad \text { for } \quad t \in H, \\
& \bar{\varphi}_{i \alpha k}(t)=f_{i \alpha}\left(t_{0}, y_{0}, u_{0}\right) \quad \text { for } \quad t \in H^{*}=q-H,
\end{aligned}
$$

and let $\bar{\varphi}_{k}(t)$ be the $\tilde{N}$-vector

$$
\varphi_{k}(t)=\left(\varphi_{i \alpha k k}(t), \alpha \in\{\alpha\}_{i}, i=1, \ldots, n\right) .
$$


For $t \in H$ and $k=k_{r} \geqslant k(\epsilon, \lambda)$, we have now

$$
\begin{gathered}
\left|t-t_{0}\right| \leqslant \nu h \leqslant \min \left[\epsilon, \delta_{0}, \delta_{0}^{\prime}\right], \\
\left|y_{k}(t)-y_{0}\right|=\left|y_{k}(t)-y_{k}\left(t_{0}\right)\right|+\left|y_{k}\left(t_{0}\right)-y\left(t_{0}\right)\right| \leqslant \epsilon+\epsilon=2 \epsilon,
\end{gathered}
$$

and, hence,

$$
\left(t, y_{k}(t)\right) \in N_{3 \epsilon}\left(t_{0}, y_{0}\right) \quad \text { for } \quad t \in H, \quad k=k_{r} \geqslant k(\epsilon, \lambda) .
$$

Thus,

$$
\begin{aligned}
& \bar{\varphi}_{k}(t)=f\left(t, y_{k}(t), u_{k}(t)\right)=\left(f_{i \alpha}\left(t, y_{k}(t), u_{k}(t)\right), \alpha \in\{\alpha\}_{i}, i=1, \ldots, n\right), \quad t \in H, \\
& \bar{\varphi}_{k}(t)=f\left(t_{0}, y_{0}, u_{0}\right)=\left(f_{i \alpha}\left(t_{0}, y_{0}, u_{0}\right), \alpha \in\{\alpha\}_{i}, i=1, \ldots, n\right), \quad t \in q-H,
\end{aligned}
$$

and, hence, for all $t \in q$, we have

$$
\widetilde{\varphi}_{k}(t) \in \widetilde{Q}\left(t_{0}, y_{0} ; 3 \epsilon\right), \quad t \in q, \quad k=k_{r} \geqslant k(\epsilon, \lambda) .
$$

Finally,

$$
h^{-v} \int_{q} \bar{\phi}_{k}(t) d t \in \operatorname{clco} \widetilde{Q}\left(t_{0}, y_{0} ; 3 \epsilon\right), \quad k=k_{r} \geqslant k(\epsilon, \lambda),
$$

since the last set is convex and closed.

Note that the relations

$$
\left|f_{i \alpha}\left(t_{0}, y_{0}, u_{0}\right)\right| \leqslant M_{1}, \quad \alpha \in\{\alpha\}_{i}, \quad i=1, \ldots, n,
$$

certainly imply that

$$
\begin{aligned}
\left|h^{-\nu} \int_{H^{*}} \bar{\varphi}_{i \alpha i k}(t) d t\right| & =\left|h^{-\nu} \int_{H^{*}} f_{i \alpha}\left(t_{0}, y_{0}, u_{0}\right) d t\right| \\
& \leqslant M_{1} h^{-\nu} \text { meas } H^{*} \leqslant M_{1} h^{-\nu}\left(h^{\nu} \eta \tilde{N}^{-1} M_{1}^{-1}\right)=\eta \tilde{N}-1
\end{aligned}
$$

with

$$
\alpha \in\{\alpha\}_{i}, \quad i=1, \ldots, n .
$$

We shall now obtain certain estimates first for $i=1, \ldots, s$ and then for $i=s+1, \ldots, n$.

Let $\chi_{\lambda q}(t), \chi_{\lambda q}^{*}(t), t \in G$, be the characteristic functions of the sets $H$ and $H^{*}$ in $G$. Since $H \cap H^{*}=\varnothing, H \cup H^{*}=q$, we have

$$
\begin{array}{ll}
\chi_{\lambda q}+\chi_{\lambda q}^{*}=1 & \text { everywhere in } q, \\
\chi_{\lambda q}=\chi_{\lambda q}^{*}=0 & \text { everywhere in } G-q .
\end{array}
$$


Then, as $k \rightarrow \infty$, hypothesis (9) implies that

$$
\begin{aligned}
& \int_{G} \chi_{\lambda q}(t) \varphi_{i \alpha k}(t) d t \rightarrow \int_{G} \chi_{\lambda Q}(t) \varphi_{i_{\alpha}}(t) d t, \\
& \int_{G} \chi_{\lambda \alpha}^{*}(t) \varphi_{i \alpha \downarrow h}(t) d t \rightarrow \int_{G} \chi_{\lambda d}^{*}(t) \varphi_{i \alpha}(t) d t,
\end{aligned}
$$

with

that is,

$$
\alpha \in\{\alpha\}_{i}, \quad i=1, \ldots, s,
$$

$$
\int_{H} \varphi_{i \alpha k} d t \rightarrow \int_{H} \varphi_{i \alpha} d t, \quad \int_{H^{*}} \varphi_{i \alpha k} d t \rightarrow \int_{H^{*}} \varphi_{i \alpha} d t .
$$

Note that the sum of these relations yields

$$
\int_{q} \varphi_{i \alpha \times k} d t \rightarrow \int_{q} \varphi_{i \alpha} d t
$$

as $k \rightarrow \infty$. We can now determine an integer $k^{\prime}=k^{\prime}\left(t_{0}, \epsilon, \lambda, \eta\right) \geqslant k(\epsilon, \lambda)$ such that, for $k=k_{r}, k \geqslant k^{\prime}\left(t_{0}, \epsilon, \lambda, \eta\right)$, we have

$$
\begin{aligned}
\left|\int_{H I} \varphi_{i \alpha k} d t-\int_{H} \varphi_{i \alpha} d t\right| & \leqslant \tilde{N}^{-1} h^{\nu} \eta \\
\left|\int_{H^{*}} \varphi_{i \alpha k} d t-\int_{Z^{*}} \varphi_{i \alpha} d t\right| & \leqslant \tilde{N}^{-1} h^{\nu} \eta,
\end{aligned}
$$

and, of course, also

$$
\left|\int_{q} \varphi_{i \alpha h} d t-\int_{q} \varphi_{i \alpha} d t\right| \leqslant 2 \tilde{N}^{-1} h^{\nu} \eta
$$

Now, for

$$
k=k_{r}, \quad k=k^{\prime}\left(t_{0}, \epsilon, \lambda, \eta\right), \quad \alpha \in\{\alpha\}_{i}, \quad i=1, \ldots, s,
$$

we have

$$
\begin{aligned}
& \left|D^{\alpha} x^{i}\left(t_{0}\right)-h^{-\nu} \int_{Q} \bar{\varphi}_{i \alpha \alpha}(t) d t\right| \\
& =\left|\varphi_{i \alpha}\left(t_{0}\right)-h^{-v} \int_{q} \varphi_{i \alpha k} d t+h^{-\nu} \int_{H^{*}} \varphi_{i \alpha k} d t-h^{-v} \int_{H^{*}} \bar{\varphi}_{i \alpha k} d t\right| \\
& \leqslant\left|\varphi_{i \alpha}\left(t_{0}\right)-h^{-\nu} \int_{Q} \varphi_{i \alpha} d t\right|+\left|h^{-v} \int_{q} \varphi_{i \alpha} d t-h^{-v} \int_{Q} \varphi_{i \alpha k} d t\right| \\
& \quad+\left|h^{-v} \int_{H^{*}} \varphi_{i \alpha k} d t-h^{-\nu} \int_{H^{*}} \varphi_{i \alpha} d t\right|+\left|h^{-\nu} \int_{H^{*}} \varphi_{i \alpha} d t\right| \\
& \quad+\left|h^{-v} \int_{H^{*}} \bar{\varphi}_{i \alpha k} d t\right|=d_{1}+d_{2}+d_{3}+d_{4}+d_{5} .
\end{aligned}
$$


By (22) we have $d_{1} \leqslant \tilde{N}^{-1} \eta$, by (33) we have $d_{2} \leqslant 2 \tilde{N}^{-1} \eta$, by (32) we have $d_{3} \leqslant \tilde{N}^{-1} \eta$, by (24) we have $d_{4} \leqslant \tilde{N}^{-1} \eta$, and by (31) we have $d_{5} \leqslant \tilde{N}^{-1} \eta$. Thus, (34) yields

$$
\left|D^{\alpha} x^{i}\left(t_{0}\right)-h^{-\nu} \int_{q} \bar{\varphi}_{i \alpha \pi k}(t) d t\right| \leqslant 6 \tilde{N}^{-1} \eta, \quad \alpha \in\{\alpha\}_{i}, \quad i=1, \ldots, s,
$$

for $k=k_{r}, k \geqslant k^{\prime}\left(t_{0}, \epsilon, \lambda, \eta\right)$.

We shall now obtain analogous estimates for $i=s+1, \ldots, n$. For these values of $i$, we have $f_{i \alpha}(t, y, u) \geqslant-c_{i \alpha}$ and, hence,

$$
\begin{aligned}
h^{-\nu} \int_{H^{*}} \varphi_{i \alpha k} d t & =h^{-\nu} \int_{H^{*}} f_{i \alpha}\left(t, y_{k}(t), u_{k}(t)\right) d t \\
& \geqslant-h^{-\nu} c_{i \alpha} \text { meas } H^{*} \geqslant-h^{-\nu} c_{i \alpha}\left(h^{\nu} \tilde{N}^{-1} c^{-1} \eta\right)>-\tilde{N}^{-1} \eta
\end{aligned}
$$

for all $k$ and where $\alpha=\alpha_{0} \in\{\alpha\}_{i}, i=s+1, \ldots, n$. Since $\alpha=\alpha_{0}=(1,1, \ldots, 1)$, we have

$$
\begin{aligned}
& h^{-\nu} \int_{q} \varphi_{i \alpha k}(t) d t=h^{-v} \Delta_{q} x_{k}^{i}, \quad i=s+1, \ldots, n, \quad k=1,2, \ldots, \\
& h^{-\nu} \int_{Q} \varphi_{i \alpha}(t) d t=h^{-v} \Delta_{q} X^{i}, \quad i=s+1, \ldots, n .
\end{aligned}
$$

On the other hand, $x^{i}=X^{i}+S^{i}$ and, by (25),

$$
h^{-v} \Delta_{q} x^{i}=h^{-v} \Delta_{q} X^{i}+h^{-v} \Delta_{q} S^{i}, \quad\left|h^{-v} \Delta_{q} S^{i}\right| \leqslant \tilde{N}^{-1} \eta
$$

Also, by force of (10), we can determine $k^{\prime}\left(t_{0}, \epsilon, \lambda, \eta\right)$ above so that, for $k=k_{r} \geqslant k^{\prime}\left(t_{0}, \epsilon, \lambda, \eta\right)$, we have

$$
\left|h^{-v} \Delta_{q} x_{z_{i}}{ }^{i}-h^{-\nu} \Delta_{q} x^{i}\right| \leqslant \tilde{N}^{-1} \eta, \quad i=s+1, \ldots, n .
$$

Finally, (37), (38), and (39) yield

$$
\begin{aligned}
& \left|h^{-\nu} \int_{q} \varphi_{i \alpha \alpha}(t) d t-h^{-\nu} \int_{q} \varphi_{i \alpha}(t) d t\right|=\left|h^{-\nu} \Delta_{q} x_{k}^{i}-h^{-\nu} \Delta_{q} X^{i}\right| \\
& \quad \leqslant\left|h^{-\nu} \Delta_{q} x_{k c}{ }^{i}-h^{-\nu} \Delta_{q} x^{i}\right|+\left|h^{-\nu} \Delta_{q} S^{i}\right| \leqslant \tilde{N}^{-1} \eta+\tilde{N}^{-1} \eta=2 \tilde{N}^{-1} \eta .
\end{aligned}
$$

For $\alpha=\alpha_{0} \in\{\alpha\}_{i}, i=s+1, \ldots, n$, we have then, as in (34),

$$
\begin{aligned}
& D^{\alpha} X^{i}\left(t_{0}\right)-h^{-v} \int_{q} \bar{\varphi}_{i \alpha k}(t) d t \\
&=\varphi_{i \alpha}\left(t_{0}\right)-h^{-v} \int_{q} \varphi_{i \alpha k} d t+h^{-v} \int_{H^{*}} \varphi_{i \alpha k} d t-h^{-\nu} \int_{H^{*}} \bar{\varphi}_{i \alpha k} d t \\
& \geqslant-\left|\varphi_{i \alpha}\left(t_{0}\right)-h^{-v} \int_{q} \varphi_{i \alpha} d t\right|-\left|h^{-v} \int_{q} \varphi_{i \alpha} d t-h^{-\nu} \int_{q} \varphi_{i \alpha k} d t\right| \\
&+h^{-v} \int_{H^{*}} \varphi_{i \alpha k k} d t-\left|h^{-\nu} \int_{H^{*}} \bar{\varphi}_{i \alpha k} d t\right| \\
&= d_{01}+d_{02}+d_{03}+d_{04} .
\end{aligned}
$$


By (26) we have $d_{01} \geqslant-\tilde{N}^{-1} \eta$, by (40) we have $d_{02} \geqslant-2 \tilde{N}^{-1} \eta$, by (36) we have $d_{03}>-\tilde{N}^{-1} \eta$, by (31) we have $d_{04}>-\tilde{N}^{-1} \eta$, and hence (41) yields

$D^{\alpha} X^{i}\left(t_{0}\right)-h^{-\nu} \int_{Q} \bar{\varphi}_{i \alpha k}(t) d t \geqslant-5 \tilde{N}^{-1} \eta, \quad \alpha=\alpha_{0} \in\{\alpha\}_{i}, \quad i=s+1, \ldots, n$,

for every $k=k_{r} \geqslant k^{\prime}\left(t_{0}, \epsilon, \lambda, \eta\right)$.

Let us denote now, for the sake of simplicity, by $y$ and $Z$ the $s$-vector $y=\left(x^{1}, \ldots, x^{s}\right)$ and $(n-s)$-vector $Z=\left(X^{s+1}, \ldots, X^{n}\right)$ respectively and, hence, by $D y$ and $D Z$ the $N$-vector and $(n-s)$-vector

$$
\begin{aligned}
& D y=\left(D^{\alpha} x^{i}, \quad \alpha \in\{\alpha\}_{i}, \quad i=1, \ldots, s\right), \\
& D Z=\left(D^{\alpha} X^{i}, \quad \alpha=\alpha_{0} \in\{\alpha\}_{i}, \quad i=s+1, \ldots, n\right) .
\end{aligned}
$$

Also, let $z_{1}$ and $z_{2}$ denote the $N$-vector and the $(n-s)$-vector

$$
\begin{aligned}
& z_{1}=\left(h^{-\nu} \int_{q} \bar{\varphi}_{i \alpha k}(t) d t, \alpha \in\{\alpha\}_{i}, i=1, \ldots, s\right), \\
& z_{2}=\left(h^{-\nu} \int_{q} \bar{\varphi}_{i \alpha k t}(t) d t, \alpha=\alpha_{0} \in\{\alpha\}_{i}, i=s+1, \ldots, n\right) .
\end{aligned}
$$

Then, relations (35) and (42) can be written in the simple form

$$
D y\left(t_{0}\right)=z_{1}+\xi_{1}, \quad D Z\left(t_{0}\right)=z_{2}+\xi_{2}+\xi_{2}{ }^{+},
$$

where $\left|\xi_{1}\right| \leqslant 6 \eta,\left|\xi_{2}\right| \leqslant 5 \eta$, and all components of $\xi_{2}{ }^{+}$are nonnegative; also, by (30), we obtain

$$
\begin{aligned}
& \left(D y\left(t_{0}\right), D Z\left(t_{0}\right)\right)=\left(z_{1}+\xi_{1}, z_{2}+\xi_{2}+\xi_{2}^{+}\right), \\
& \left(z_{1}, z_{2}\right)=h^{-v} \int_{q} \bar{\varphi}_{k}(t) d t \in \operatorname{cl} \operatorname{co} \tilde{Q}\left(t_{0}, y_{0} ; 3 \epsilon\right) .
\end{aligned}
$$

Since all components of $\xi_{2}{ }^{+}$are nonnegative and because of the particular definition of $\mathscr{Q}$, we have also

$$
\left(z_{1}, z_{2}+\xi_{2}^{+}\right) \in \operatorname{cl} \operatorname{co} \widetilde{Q}\left(t_{0}, y_{0} ; 3 \epsilon\right)
$$

and, hence,

$$
\left(z_{1}+\xi_{1}, z_{2}+\xi_{2}+\xi_{2}^{+}\right) \in\left(\operatorname{cl} \operatorname{co} \widetilde{Q}\left(t_{0}, y_{0} ; 3 \epsilon\right)\right)_{12 \eta},
$$

or

$$
\left(D y\left(t_{0}\right), D Z\left(t_{0}\right)\right) \in\left(\mathrm{cl} \operatorname{co} \widetilde{Q}\left(t_{0}, y_{0} ; 3 \epsilon\right)\right)_{12 \eta} .
$$


Here, $t_{0}$ is a fixed point and, hence,

$$
\begin{gathered}
\left(D y\left(t_{0}\right), D Z\left(t_{0}\right)\right) \in \bigcap_{\eta>0}\left(\operatorname{cl} \operatorname{co} \widetilde{Q}\left(t_{0}, y_{0} ; 3 \epsilon\right)\right)_{12 \eta}, \\
\left(D y\left(t_{0}\right), D Z\left(t_{0}\right)\right) \in \mathrm{cl} \operatorname{co} \widetilde{Q}\left(t_{0}, y_{0} ; 3 \epsilon\right),
\end{gathered}
$$

since the last set is closed. Also,

$$
\left(D y\left(t_{0}\right), D Z\left(t_{0}\right)\right) \in \bigcap_{\epsilon>0} \operatorname{cl} \operatorname{co} \check{Q}\left(t_{0}, y_{0} ; 3 \epsilon\right)=Q\left(t_{0}, y_{0}\right)
$$

with $y_{0}=y\left(t_{0}\right)$, because of property $(Q)$. Here, $t_{0}$ is an arbitrary point of $C_{\lambda}^{\prime}$. Hence,

$$
(D y(t), D Z(t)) \in \widetilde{Q}(t, y(t))
$$

for all points $t \in G^{\prime \prime}=\cup_{\lambda} C_{\lambda}^{\prime}$ and, by (21), meas $G^{\prime \prime}=$ meas $G$. Thus, for almost all $t \in G$, there is some $\tilde{u}(t) \in U(t, y(t))$ such that

$$
\begin{aligned}
D^{\alpha} X^{i}(t) & =f_{i \alpha}(t, y(t), \bar{u}(t)), \quad \alpha \in\{\alpha\}_{i}, \quad i=1, \ldots, s, \\
D^{\alpha} X^{i}(t) & =f_{i \alpha}(t, y(t), \bar{u}(t)), \quad \alpha=\alpha_{0} \in\{\alpha\}_{i}, \quad i=s+1, \ldots, n .
\end{aligned}
$$

The existence of at least one measurable function $u(t)$ as above follows by the same argument used for $\nu=1$ in Ref. 2, Section 5 (for a general statement to the same effect, see also Ref. 9). Closure Theorem 2 is hereby proved.

3.4. Remark. We mention here variants of Closure Theorems 1 and 2 which are of some interest. One of these actually will be applied in Section 5. We may assume, for instance, that $G$ is made up of components $G_{1}, \ldots, G_{\mu}$ and that, in each of these, there is a different system of $\{\alpha\}_{i}, i=1, \ldots, s$, and of functions $f_{i \alpha}$. Another situation is of interest. Assume that $G_{\sigma}$, $\sigma=1, \ldots, \mu$, are finitely many open bounded subsets of $E_{v}$ and that, for each $G_{\sigma}$, there is a given set $\{\alpha\}_{i \sigma}, i=1, \ldots, n, \sigma=1, \ldots, \mu$, and a system $f_{i \alpha}$ of functions $f$. Now, let us consider all possible nonempty intersections $F_{r}=G_{\sigma_{1}} \cap G_{\sigma_{2}} \cap \cdots \cap G_{\sigma_{p}}$ of $p$ of the sets $G_{\sigma}, 1 \leqslant p \leqslant \mu$. These sets $F_{r}, r=1, \ldots, N$, are finitely many and each is a nonempty bounded open subset of $E_{v}$. For each of these $N$ sets $F_{r}=G_{\sigma_{1}} \cap \cdots \cap G_{\sigma_{p}}$, we shall consider the vector function $f^{(r)}(t, x, u)$ whose components are all those of the functions $f=\left(f_{i a}\right), \alpha \in\{\alpha\}_{i v}, i=1, \ldots, n, \sigma=\sigma_{1}, \ldots, \sigma_{p}$. We shall then require that, for each $r$, the set $Q^{(r)}(t, x)$ corresponding to $f^{(r)}$ is a convex subset of the relative Euclidean space $E_{M_{q}+(n-s)}$. Here, $M_{r}$ is the total number of all distinct indices $\alpha \in\{\alpha\}_{i o}, i=1, \ldots, n, \sigma=\sigma_{1}, \ldots, \sigma_{p}$. In other words, in each 
$G_{\sigma}$, we have a different system of partial differential equations $D^{\alpha} x^{i}=f_{i \alpha}$ and, in each nonempty intersection $F_{r}=G_{\sigma_{1}} \cap \cdots \cap G_{\sigma_{o}}$, we consider the logical union of the various differential systems. We assume that these systems are compatible, and then the hypothesis that $\widetilde{Q}^{(r)}(t, x)$ is convex corresponds to the usual hypothesis for these composite differential systems. A further extension can be obtained by assuming that the functions $f_{i \alpha}$ are sectionally continuous in each set $G_{\sigma}$ but, for instance, coincide on each set $F_{r}$ with functions which are continuous on the closure of $F_{r}$. These variants of Closure Theorems 1 and 2 are proved exactly by the same argument.

\section{More Notations for the Existence Theorems}

We shall use the same general notations as in Section 2. Besides the $N$-vector $f(t, x, u)=\left(f_{i \alpha}\right)$, we now consider a scalar function $f_{0}(t, x, u)$ continuous on $M$, and we shall denote by $\tilde{f}(t, x, u)$ the $(N+1)$-vector function $f(t, x, u)=\left(f_{0}, f_{i \alpha}\right)$ continuous on $M$. Concerning the $n$-vector functions $x(t)=\left(x^{1}, \ldots, x^{n}\right), t \in G$, we shall require that each component $x^{i}(t), t \in G$, belongs to a Sobolev class $W_{p_{i}}^{t_{i}}(G)$ for given $l_{i}$ and $p_{i}, 1 \leqslant l_{i} \leqslant l$, $p_{i}>1, i=1,2, \ldots, n$. By force of Sobolev's imbedding theorems (Ref. 4), each function $x^{i}$ and each derivative $D^{\alpha} x^{i}$ of order $\alpha=\left(\alpha_{1}, \ldots, \alpha_{\nu}\right)$, $0 \leqslant|\alpha| \leqslant l_{i}-1$, has boundary values $\varphi_{\alpha}{ }^{i}$ defined almost everywhere on the boundary $S=\partial G$ of $G$, and each $\varphi_{\alpha}{ }^{i}$ is of class $L_{p_{i}}$ on $S$.

We shall now require a set $(B)$ of boundary conditions involving the boundary values of the functions $x^{i}$ and their derivatives $D^{\alpha} x^{i}$, $0 \leqslant|\alpha| \leqslant l_{i}-1$. On the boundary conditions $(B)$, we assume only the following closure property $\left(P_{1}\right):$ If $x(t)=\left(x^{1}, \ldots, x^{n}\right), x_{k}(t)=\left(x_{k}{ }^{1}, \ldots, x_{k}{ }^{n}\right)$, $t \in G, k=1,2, \ldots$, are vector functions whose components $x^{i}, x_{k}{ }^{i}$ belong to the Sobolev class $W_{p_{i}}^{l_{i}}(G)$, if $D^{\beta} x_{k}{ }^{i}(t) \rightarrow D^{\beta} x^{i}(t)$ as $k \rightarrow \infty$ strongly in $L_{p_{i}}(G)$ for every $\beta$ with $0 \leqslant|\beta| \leqslant l_{i}-1$, if $D^{\beta} x_{k}^{i}(t) \rightarrow D^{\beta} x^{i}(t)$ as $k \rightarrow \infty$ weakly in $L_{p_{i}}(G)$ for every $\beta$ with $|\beta|=l_{i}$, and if the boundary values $\varphi_{k \alpha}^{i}$ of $x_{k}^{i}(t)$, $i=1, \ldots, n, 0 \leqslant|\alpha| \leqslant l_{i}-1$, on $\partial G$ satisfy the boundary conditions $(B)$, then the boundary values $\varphi_{\alpha}{ }^{i}$ of $x^{i}(t), i=1, \ldots, n, 0 \leqslant|\alpha| \leqslant l_{i}-1$, on $\partial G$ satisfy the boundary conditions $(B)$.

For instance, if the boundary conditions $(B)$ are defined by stating that some of the boundary values $\varphi_{k \alpha}^{i}(t)$ coincide with preassigned continuous functions $\varphi_{\alpha}{ }^{i}$ on certain parts of $S=\partial G$, then, by force of Sobolev's imbedding theorems (Ref. 4), we know that property $\left(P_{1}\right)$ is valid.

We shall need a further property of boundary conditions $(B)$, say $\left(P_{2}\right)$ : If $x(t)=\left(x^{1}, \ldots, x^{n}\right), t \in G$, denotes any vector function satisfying boundary 
conditions $(B)$, whose components $x^{i}(t) \in W_{p_{i}}^{l_{i}}(G), \quad p_{i}>1, \quad 1 \leqslant l_{i} \leqslant l$, satisfy

$$
\int_{G}\left|D^{\beta} x^{i}(t)\right|^{p_{i}} d t \leqslant M_{i \beta}
$$

for all $\beta=\left(\beta_{1}, \ldots, \beta_{\nu}\right)$ with $|\beta|=l_{i}, i=1, \ldots, n$, and constants $M_{i \beta}$, then there are constants $M_{i x}$ such that

$$
\int_{G}\left|D^{\alpha} x^{i}(t)\right|^{p_{i}} d t \leqslant M_{i \propto}
$$

for all $\alpha=\left(\alpha_{1}, \ldots, \alpha_{\nu}\right)$ with $0 \leqslant|\alpha| \leqslant l_{i}-1, i=1, \ldots, n$, where the constants $M_{i \propto}$ depend only on $p_{i}, \nu$, all $M_{i \beta}, G$, and boundary conditions $(B)$, but not on the vector function $x(t)$ above.

For instance, the boundary conditions $(B)$ defined by preassigning the continuous boundary value functions $\varphi_{\alpha}^{i}$ on $\partial B$ of all derivatives $D^{\alpha} x^{i}(t)$, $\alpha=\left(\alpha_{1}, \ldots, \alpha_{v}\right), 0 \leqslant|\alpha| \leqslant l_{i}-1, i=1, \ldots, \nu$, satisfy condition $\left(P_{2}\right)$.

A pair $x(t)=\left(x^{1}, \ldots, x^{n}\right), u(t)=\left(u^{1}, \ldots, u^{m}\right), t \in G$, with $x^{i} \in W_{p_{i}}^{l_{i}}(G)$, $u^{j}$ measurable in $G$, satisfying $(t, x(t)) \in A, u(t) \in U(t, x(t)), D^{\alpha} x^{i}(t)=f_{i \alpha}(t, x(t)$, $u(t)), \alpha \in\{\alpha\}_{i}, i=1, \ldots, n$, a.e. in $G$, and $f_{0}(t, x(t), u(t)) \in L_{1}(G)$, is said to be admissible. A class $\Omega$ of admissible pairs is said to be complete if, for any sequence $x_{k}, u_{k}, k=1,2, \ldots$, of pairs all in $\Omega$ and any admissible pair $x, u$ such that $x_{k} \rightarrow x$ in the sense described under $P_{1}$, the pair $x, u$ belongs to $\Omega$. The class of all admissible pairs is obviously complete.

\section{Existence Theorems}

5.1. Existence Theorem 1. Let $G$ be a bounded open set of some class $K_{l}, l \geqslant 1$, of the $t$-space $E_{y}$, for every $t \in \mathrm{cl} G$ let $A(t)$ be a nonempty subset of the $x$-space $E_{n}$, and assume that the set $A$ of all $(t, x) \in E_{\nu} \times E_{n}$ with $t \in \operatorname{cl} G, x \in A(t)$, is closed. For every $(t, x) \in A$, let $U(t, x)$ be a nonempty closed subset of the $u$-space $E_{m}$, and assume that $U(t, x)$ satisfies property $(U)$ in $A$. Let $M$ be the set of all $(t, x, u) \in E_{v} \times E_{n} \times E_{m}$ with $(t, x) \in A$ and $u \in U(t, x)$. For every $i=1, \ldots, n$, let $\{\alpha\}_{i}$ be a given finite system of indices $\alpha=\left(\alpha_{1}, \ldots, \alpha_{v}\right), 0 \leqslant|\alpha| \leqslant l_{i} \leqslant l$, and let $N$ be the total number of elements $\alpha \in\{\alpha\}_{i}, i=1, \ldots, n$. Let $\tilde{f}(t, x, u)=\left(f_{0}, f_{i \alpha}\right.$, $\left.\alpha=\{\alpha\}_{i}, i=1, \ldots, n\right)=\left(f_{0}, f\right)$ be a continuous $(N+1)$-vector function on $M$ and assume that the set $\tilde{Q}(t, x)=\tilde{f}(t, x, U(t, x))$ is a convex closed subset of $E_{N+1}$ for every $(t, x) \in A$ and satisfies property $(Q)$ in $A$. Let $(B)$ be a system of boundary conditions satisfying properties $\left(P_{1}\right)$ and $\left(P_{2}\right)$. 
Let $\Omega$ be a nonempty complete class of pairs $x(t)=\left(x^{1}, \ldots, x^{n}\right), u(t)=\left(u^{1}, \ldots, u^{m}\right)$, $t \in G, x^{i}(t) \in W_{p_{i}}^{l_{i}}(G), 1 \leqslant l_{i} \leqslant l, p_{i}>1, i=1, \ldots, n, u^{j}(t)$ measurable in $G$, $j=1, \ldots, m$, satisfying (a) the constraints

$$
(t, x(t)) \in A, \quad u(t) \in U(t, x(t)) \quad \text { a.e. in } G,
$$

(b) the system of partial differential equations

$$
D^{\alpha} x^{i}(t)=f_{i \alpha}(t, x(t), u(t)) \quad \text { a.e. in } G, \quad \alpha \in\{\alpha\}_{i}, \quad i=1, \ldots, n,
$$

(c) the boundary conditions $(B)$ on the boundary $\partial G$ of $G$ concerning the boundary values of the functions $x^{i}(t)$ and their generalized partial derivatives $D^{\beta} x^{i}(t)$ of orders $\beta, 0 \leqslant|\beta| \leqslant l_{i}-1, i=1, \ldots, n$, and (d) the system of inequalities

$$
\begin{gathered}
\int_{G}\left|D^{\xi^{2}} x^{i}(t)\right|^{p_{i}} d t \leqslant N_{i \beta} \text { for all } \beta \text { with }|\beta|=l_{i}, \quad i=1, \ldots, n, \\
\int_{G}\left|f_{0}(t, x(t), u(t))\right|^{p_{0}} d t \leqslant N_{0}
\end{gathered}
$$

where $N_{i \beta}, N_{0}$ are given constants, and $p_{0}, p_{i}>1$ are given. Then, the cost functional

$$
I[x, u]=\int_{G} f_{0}(t, x(t), u(t)) d t
$$

possesses an absolute minimum and an absolute maximum in $\Omega$.

5.2. Proof. Let $R$ be an interval $R=[a, b]$ containing $\mathrm{cl} G$ in its interior, say cl $G \subset \operatorname{int} R \subset E_{\nu}$. It is not restrictive to assume $R=[0, b]$ where 0 and $b$ represent the $v$-vectors $(0, \ldots, 0)$ and $(b, \ldots, b)$. Let $x^{0}$ denote a new variable and $x=\left(x^{0}, x\right)=\left(x^{0}, x^{1}, \ldots, x^{n}\right)$. Let $l_{0}=\nu$ and let $\vec{f}_{0}(t, x, u)$ be a new scalar function defined by

$$
\begin{aligned}
& \tilde{f}_{0}(t, x, u)=0 \quad \text { for } \quad t \in R-\operatorname{cl} G, \quad x \in E^{n}, \quad u \in E^{m}, \\
& f_{0}(t, x, u)=f_{0}(t, x, u) \quad \text { for } \quad t \in \operatorname{cl} G, \quad(t, x, u) \in M .
\end{aligned}
$$

We shall denote by $x^{0}(t), t \in R$, a new scalar function which is $L$-integrable in $R$, possesses the generalized partial derivative $\partial^{v} x^{0} / \partial t^{1} \cdots \partial t^{\nu}$, or $D^{\alpha} x^{0}$ with $\alpha=\alpha_{0}=(1, \ldots, 1)$, which is also $L_{1}$-integrable in $R$, satisfies the partial differential equation

$$
D^{\alpha} x^{0}=f_{0}(t, x(t), u(t)) \quad \text { a.e. in } R, \quad \alpha=(1, \ldots, 1),
$$


and the boundary conditions

$$
x^{0}\left(t_{i}^{\prime}, 0\right)=0, \quad\left(t_{i}^{\prime}, 0\right) \in \partial R, \quad i=1, \ldots, \nu
$$

where $t_{i}^{\prime}$ denotes the $(v-1)$-vector $t_{i}^{\prime}=\left(t_{1}, \ldots, t_{i-1}, t_{i+1}, \ldots, t_{v}\right)$. Then, we have

$$
\begin{aligned}
& x^{0}(b)=\int_{R} \tilde{f}_{0}(t, x(t), u(t)) d t=\int_{G} f_{0}(t, x(t), u(t)) d t=I[x, u], \\
& x^{0}(t)=\int_{0}^{t} \tilde{f}_{0}(\tau, x(\tau), u(\tau)) d \tau, \quad t \in R,
\end{aligned}
$$

where the last integral is taken over the interval $\left[0 \leqslant \tau^{i} \leqslant t^{i}, i=1, \ldots, \nu\right]$, and then

$$
\int_{R}\left|D^{\alpha} x^{0}(t)\right|^{p_{0}} d t \leqslant N_{0}, \quad \alpha=\alpha_{0}=(1, \ldots, 1) .
$$

Also, if $q_{0}^{-1}+p_{0}^{-1}=1$, then

$$
\left|x^{0}(b)\right|=|I[x, u]| \leqslant\left(\int_{R} d t\right)^{1 / q_{0}}\left(\int_{R}\left|f_{0}\right|^{p_{0}} d t\right)^{1 / p_{0}} \leqslant b^{\nu / a_{0}} N_{0}^{1 / p_{0}}
$$

and thus $I[x, u]$ is bounded above and below in $\Omega$. As usual, we shall use the vector variable $\tilde{x}=\left(x^{0}, x\right)$. If $i$ denotes the infimum of $I[x, u]$ in $\Omega$, let $x_{k}, u_{k}$, $k=1,2, \ldots$, be a sequence of pairs all in $\Omega$ with $I\left[x_{k}, u_{k}\right]=x_{k}{ }^{0}(b) \rightarrow i$ as $k \rightarrow \infty$. By the weak compactness of the unit ball in $W_{p_{i}}^{t_{i}}(G), i=1, \ldots, n$, and in $L_{p_{0}}(G)$, there exists a subsequence, say still $\left[x_{k}, u_{k}\right]$, and limit functions $x=\left(x^{1}, \ldots, x^{n}\right)$ and $\psi$, such that, as $k \rightarrow \infty$, we have

$$
\begin{aligned}
D^{\alpha}{\mathcal{X}_{k}}_{k}{ }^{i} & \rightarrow D^{\alpha} x^{i} \text { weakly in } L_{p_{i}}(G), \quad \alpha \in\{\alpha\}_{i}, \quad i=1, \ldots, n, \\
x_{k}{ }^{i} & \rightarrow x^{i} \text { strongly in } L_{p_{i}}(G), \quad i=1, \ldots, n, \\
D^{\alpha} x_{k i}{ }^{0} & \rightarrow \psi \text { weakly in } L_{p_{0}}(G), \quad \alpha=\alpha_{0}=(1, \ldots, 1) .
\end{aligned}
$$

If $x^{0}(t), t \in G$, is defined by

$$
x^{0}(t)=\int_{0}^{t} \psi(\tau) d \tau,
$$

where the integration is taken in the interval $[0, t]$, or $0 \leqslant \tau^{i} \leqslant t^{i}, i=1, \ldots, \nu$, then

$$
\psi(t)=D^{\alpha} x^{0}(t) \quad \text { a.e. in } G, \quad \alpha=\alpha_{0}=(1, \ldots, 1)
$$


and since $D^{\alpha} x_{k}^{0}=\bar{f}_{0}$ is zero in $R-\mathrm{cl} G$, we know that also $\psi=0$ in $R-\mathrm{cl} G$. Finally, the weak convergence $D^{\alpha} x_{k}{ }^{0} \rightarrow D^{\alpha} x^{0}$ in $L_{p_{0}}(R), \alpha=\alpha_{0}=$ $(1, \ldots, 1)$, implies that, as $k \rightarrow \infty$,

$$
\int_{R} \chi_{t}(\tau) D^{\alpha} x_{k}^{0}(\tau) d \tau \rightarrow \int_{R} \chi_{t}(\tau) D^{\alpha} x^{0}(\tau) d \tau
$$

where $\chi_{t}(\tau)$ is the characteristic function of the interval $[0, t]$ in $R$. In other words, as $k \rightarrow \infty$,

$$
\int_{0}^{t} D^{\alpha} x_{k}^{0}(\tau) d \tau \rightarrow \int_{0}^{t} D^{\alpha} x^{0}(\tau) d \tau, \quad \alpha=\alpha_{0}=(1, \ldots, 1)
$$

or $x_{k}{ }^{0}(t) \rightarrow x^{0}(t)$ pointwise as $k \rightarrow \infty$ for every $t \in R$. We can now apply Closure Theorem 1 , with pointwise convergence $x_{k}{ }^{p} \rightarrow x^{0}$ replacing strong convergence, with $R$ replacing $G$ for the component $x^{0}$ as in the remark at the end of Section 3, and with $\vec{f}_{0}$ continuous in $G$ and in $R-\mathrm{cl} G$, precisely, sectionally continuous in $R$. Thus, by Closure Theorem 1 , there exists a measurable function $u(t)=\left(u^{1}, \ldots, u^{m}\right), t \in G$, such that

$$
\begin{gathered}
(t, x(t)) \in A, \quad u(t) \in U(t, x(t)) \quad \text { a.e. in } G, \\
D^{\alpha} x^{i}=f_{i \alpha}(t, x(t), u(t)) \quad \text { a.e. in } G, \quad \alpha \in\{\alpha\}_{i}, \quad i=1, \ldots, n, \\
D^{\alpha} x^{0}=\tilde{f}_{0}(t, x(t), u(t)) \quad \text { a,e. in } R_{3} \quad \alpha=\alpha_{0} .
\end{gathered}
$$

Hence,

$$
x^{0}(b)=I[x, u]=\int_{R} \vec{f}_{0} d t=\int_{G} f_{0} d t=\lim _{k \rightarrow \infty} x_{k}^{0}(b)=i
$$

and the pair $x, u$ belongs to $\Omega$, because $\Omega$ is complete. This proves that $I[x, u]$ has an absolute minimum in $\Omega$. The same proof holds for the maximum.

5.3. Existence Theorem 2, Let $G$ be a bounded open set of class $K_{l}, l>1$, of the $t$-space $E_{v}$, for every $t \in \mathrm{cl} G$ let $A(t)$ be a nonempty subset of the $x$-space $E_{n}$, and assume that the set $A$ of all $(t, x) \in E_{\nu} \times E_{n}$ with $t \in \mathrm{cl} G, x \in A(t)$, is closed. For every $(t, x) \in A$, let $U(t, x)$ be a nonempty closed subset of the $u$-space $E_{m}$, and assume that $U(t, x)$ satisfies property $(U)$ in $A$. Let $M$ be the set of all $(t, x, u) \in E_{v} \times E_{n} \times E_{m}$ with $(t, x) \in A$, $u \in U(t, x)$. For every $i=1, \ldots, n$, let $\{\alpha\}$, be a given finite system of indices $\alpha=\left(\alpha_{1}, \ldots, \alpha_{w}\right), 0 \leqslant|\alpha| \leqslant l_{i} \leqslant l$, and let $N$ be the total number of elements $\alpha \in\{\alpha\}_{i}, i=1, \ldots, n$. Let $\tilde{f}(t, x, u)=\left(f_{0}, f_{i \alpha}, \alpha \in\{\alpha\}_{i}, i=1, \ldots, n\right)=\left(f_{0}, f\right)$ 
be a continuous $(N+1)$-vector function on $M$, and assume that the set $\widetilde{Q}(t, x)$ of all $z=\left(z^{0}, z^{1}, \ldots, z^{n}\right)=\left(z^{0}, z\right) \in E_{N+1}$ with $z^{0} \geqslant f_{0}(t, x, u), z=$ $f(t, x, u), u \in U(t, x)$ is a convex closed subset of $E_{N+1}$ for every $(t, x) \in A$ and satisfies property $(Q)$ in $A$. Also, assume that $f_{0}(t, x, u) \geqslant-M_{0}$ for all $(t, x, u) \in M$ and some constant $M_{0} \geqslant 0$. Let $(B)$ be a system of boundary conditions satisfying properties $\left(P_{1}\right)$ and $\left(P_{2}\right)$. Let $\Omega$ be a nonempty complete class of pairs $x(t)=\left(x^{1}, \ldots, x^{n}\right), u(t)=\left(u^{1}, \ldots, u^{m}\right), \quad t \in G, x^{i}(t) \in W_{p_{i}}^{l_{i}}(G)$, $1 \leqslant l_{i} \leqslant l, p_{i}>1, i=1, \ldots, n, u^{j}(t)$ measurable in $G, j=1, \ldots, m$, satisfying (a) the constraints

$$
(t, x(t)) \in A, \quad u(t) \in U(t, x(t)) \quad \text { a.e. in } G,
$$

(b) the system of partial differential equations

$$
D^{\alpha} x^{i}(t)=f_{i \propto}(t, x(t), u(t)) \quad \text { a.e. in } G, \quad \alpha \in\{\alpha\}_{i}, \quad i=1, \ldots, n,
$$

(c) the boundary conditions $(B)$ on the boundary $\partial G$ of $G$ concerning the boundary values of the functions $x^{i}(t)$ and their generalized partial derivatives $D^{\beta} x^{i}(t)$ of orders $\beta, 0 \leqslant|\beta| \leqslant l_{i}-1, i=1, \ldots, n$, and (d) the system of inequalities

$$
\int_{G}\left|D^{\beta} x^{i}(t)\right|^{p_{i}} d t \leqslant N_{i \beta}
$$

for all $\beta$ with $|\beta|=l_{i}, \beta \notin\left\{\alpha_{i}, i=1,2, \ldots, n\right.$, where $N_{i \beta}$ are given constants. Assume that $f_{0}(t, x(t), u(t))$ is $L_{1}$-integrable in $G$. Finally, assume that whenever

$$
\int_{G} f_{0}(t, x(t), u(t)) d t \leqslant L_{0}
$$

for some constants $L_{0}$ and pairs $x(t), u(t)$ in $\Omega$, then for the same pairs we have also

$$
\int_{G}\left|D^{\alpha} x^{i}(t)\right|^{p_{i}} d t \leqslant L_{i \alpha}, \quad \alpha \in\{\alpha\}_{i}, \quad i=1, \ldots, n,
$$

for constants $L_{i \alpha}$ depending only on $L_{0}, \Omega, p_{i}, l_{i}$ on the boundary conditions $(B)$, but not on the particular pair $x, u$. Then, the cost functional

$$
I[x, u]=\int_{G} f_{0}(t, x(t), u(t)) d t
$$

possesses an absolute minimum in $\Omega$. If, for given $i$ and $\beta$ as in (d), it happens that, for any $L_{0} \geqslant 0$ sufficiently large, there is some $N_{i \beta}$ such that $I[x, u] \leqslant L_{0}$ implies $\int_{G}\left|D^{\beta} x^{i}(t)\right|^{p_{i}} d t \leqslant N_{i \beta}$, then the absolute minimum still exists, even if in defining $\Omega$ we disregard the corresponding requirement in (d); the absolute minimum, however, may change. 
5.4. Proof. By hypothesis, we have $f_{0}(t, x, u)+M_{0} \geqslant 0$ for all $(t, x, u) \in M$. For every pair $x, u$ of the class $\Omega$, we have now

$$
+\infty>I[x, u]=\int_{G} f_{0} d t \geqslant-M_{0} \text { meas } G>-\infty .
$$

Then, the infimum $i$ of $I[x, u]$ in the class $\Omega$ is finite. Let $x_{k}(t), u_{f k}(t), t \in G$, $k=1,2, \ldots$, be a sequence of pairs all in $\Omega$ such that $I\left[x_{k}, u_{k}\right] \rightarrow i$ as $k \rightarrow \infty$. We may assume

$$
i \leqslant I\left[x_{k}, u_{k}\right]=\int_{G} f_{0}\left(t, x_{k}(t), u_{k}(t)\right) d t \leqslant i+k^{-1} \leqslant i+1, \quad k=1,2, \ldots
$$

Then, by hypothesis, there are constants $L_{i \alpha}$ such that

$$
\int_{G}\left|D^{\alpha} x_{k}^{i}(t)\right|^{p_{i}} d t \leqslant L_{i \alpha}, \quad \alpha \in\{\alpha\}_{i}, \quad i=1, \ldots, n, \quad k=1,2, \ldots,
$$

and, by (d),

$\int_{G}\left|D^{\beta} x_{k}^{i}(t)\right|^{p_{i}} d t \leqslant N_{i \beta}$ for all $|\beta|=l_{i}, \quad \beta \notin\{\alpha\}_{i}, i=1, \ldots, n, \quad k=1,2, \ldots$

Relations (45) and (46) show that the sequence $\left[x_{k}{ }^{i}\right]$ belongs to a well determined balls of the Sobolev space $W_{p_{i}}^{l_{i}}(G), i=1, \ldots, n$. By the weak compactness of such balls, there is a subsequence, say still $\left[x_{k}\right]$ for the sake of simplicity, converging weakly toward a vector function $x(t)=\left(x^{1}, \ldots, x^{n}\right), t \in G$, with $x^{i} \in W_{p_{i}}^{l_{i}}(G)$, and, precisely, by Sobolev's imbedding theorems (Ref. 4),

$$
\begin{array}{ll}
D^{\alpha} x_{k}^{i} \rightarrow D^{\alpha} x^{i} \text { strongly in } L_{p_{i}}(G), & |\alpha| \leqslant l_{i}-1, \quad i=1, \ldots, n, \\
D^{\alpha} x_{k^{i}}{ }^{i} \rightarrow D^{\alpha} x^{i} \text { weakly in } L_{p_{i}}(G), & |\alpha|=l_{i}, \quad i=1, \ldots, n .
\end{array}
$$

By properties $\left(P_{1}\right)$ and $\left(P_{2}\right)$, we conclude that $x$ satisfies the boundary conditions $(B)$ on $\partial G$.

Let $R$ be an interval containing $\mathrm{cl} G$ in its interior, $G \subset \mathrm{cl} G \subset \operatorname{int} R \subset R$. We can assume $R=[0, b]$, where 0 and $b$ denote $(0, \ldots, 0)$ and $(b, \ldots, b)$. Let $\varphi_{k}(t)=f_{0}\left(t, x_{k}(t), u_{k}(t)\right)$ for $t \in G, \varphi_{k}(t)=0$ for $t \in R-G$, and let $\varphi^{-}(t)=-M_{0}, t \in R$, and $\varphi_{k}{ }^{+}(t)=\varphi_{k}(t)+M_{0}, t \in R$, so that $\varphi_{k}{ }^{+}+\varphi_{k}{ }^{-}=$ $\varphi_{k}, \varphi_{k}{ }^{+}(t) \geqslant 0, \varphi^{-}(t) \leqslant 0$ everywhere in $R$. Let $x_{k}{ }^{0}, x_{k}{ }^{+}, x^{-}$be the functions

$$
x_{k}{ }^{0}(t)=\int_{0}^{t} \varphi_{k}(\tau) d \tau, \quad x_{k}{ }^{+}=\int_{0}^{t} \varphi_{k}{ }^{+}(\tau) d \tau, \quad x-(t)=\int_{0}^{t} \varphi^{-}(\tau) d \tau, \quad t \in R,
$$


where the integrals range on the interval $[0, t]$, or $0 \leqslant \tau^{i} \leqslant t^{i}, i=1, \ldots, \nu$. Hence,

$$
\begin{aligned}
& x_{k}^{0}(b)=\int_{R} \varphi_{k}(t) d t=\int_{G} \varphi_{k}(t) d t=I\left[x_{k}, u_{k}\right] \leqslant i+1, \\
& x_{k}^{0}(t)=x^{-}(t)+x_{k}^{+}(t), \quad t \in R, \quad k=1,2, \ldots
\end{aligned}
$$

For every integral $I \subset R$, we denote as usual by $\Delta_{I} x_{k}^{0}, \Delta_{I} x_{k}^{+}, \Delta_{I} x^{-}$the differences of order $v$ of the indicated functions relative to the $2^{\nu}$ vertices of $I$, and we have $\Delta_{I} x_{k}^{0}=\Delta_{I^{x}}{ }^{+}+\Delta_{I} x^{-}$, with

$\Delta_{I} x_{k}^{0}=\int_{I} \varphi_{k}(t) d t, \quad \Delta_{I} x_{k}{ }^{+}=\int_{I} \varphi_{k}{ }^{+}(t) d t, \quad \Delta_{I} x^{-}=\int_{I} \varphi^{-(t) d t}=-M_{0}$ meas $I$.

If $t=\left(t^{1}, \ldots, t^{\nu}\right), t^{\prime}=\left(t^{\prime}, \ldots, t^{\prime}\right)$ are any two points of $R$, then obviously $\left|t^{i}-t^{\prime}\right| \leqslant\left|t-t^{\prime}\right|, i=1, \ldots, \nu$. Also, we denote by $t_{0}=t_{1}, t_{2}, \ldots, t_{v}=t^{\prime}$, the points of $R$ defined by $t_{j}=\left(t^{1}, \ldots, t^{\nu-j}, t^{\prime \nu-j+1}, \ldots, t^{\prime} \nu\right), j=0,1, \ldots, \nu$, and by $r_{j}$ the intervals

$r_{j}=\left[0, t^{1}\right] \times \cdots \times\left[0, t^{\nu-j+1}\right] \times\left[t^{\nu-j}, t^{\prime y-j}\right] \times\left[0, t^{\prime y-j+1}\right] \times \cdots \times\left[0, t^{\prime} v\right.$,

$$
j=0,1, \ldots, v-1 \text {. }
$$

Then,

$$
x_{k i}^{0}(t)-x_{k}^{0}\left(t^{\prime}\right)=\Sigma_{j}\left[x_{k}^{0}\left(t_{j}\right)-x_{k}^{0}\left(t_{j+1}\right)\right],
$$

where $\Sigma$ ranges over all $j=0,1, \ldots, \nu-1$ and

$$
x_{k}^{0}\left(t_{j}\right)-x_{k}^{0}\left(t_{j+1}\right)=\int_{r_{j}} \varphi_{k}(t) d t
$$

and analogous formulas hold for $x_{k}{ }^{+}$and $x^{-}$. Since

$$
\text { meas } r_{j} \leqslant b^{\nu-1}\left|t^{j}-t^{\prime j}\right| \leqslant b^{\nu-1}\left|t-t^{\prime}\right|
$$

and $\varphi^{-}=-M_{0}$, we have

$$
\left|x-(t)-x^{-}\left(t^{\prime}\right)\right| \leqslant v M_{0} b^{y-1}\left|t-t^{\prime}\right|, \quad t, t^{\prime} \in R .
$$

On the other hand, $\varphi_{k}{ }^{+} \geqslant 0$ and, hence, $\Delta_{r} x_{k}{ }^{+} \geqslant 0$ for every $I \subset R$, $k==1,2, \ldots$. 
If $\left[I_{1}, \ldots, I_{L}\right]$ denotes any finite subdivision of $R$ into nonoverlapping intervals $I_{s}, s=1, \ldots, L$, then

$$
\begin{aligned}
\Sigma_{s}\left|\Delta_{I_{s}} x^{-}\right| & =-\Sigma_{s} \Delta_{I_{s}} x^{-}=M_{0} \Sigma_{s} \text { meas } I_{s}=M_{0} b^{v}, \\
\Sigma_{s}\left|\Delta_{I_{s}} x_{k}{ }^{+}\right| & =\Sigma_{s} \Delta_{I_{s}} x_{k}{ }^{+}=\Sigma_{s} \Delta_{I_{s}} x_{k}{ }^{0}-\Sigma_{s} \Delta_{I_{s}} x^{--}=x_{k}^{0}(b)+M_{0} b^{\nu} \leqslant i+1+M_{0} b^{\nu},
\end{aligned}
$$

and, thus,

$$
\Sigma_{s}\left|\Delta_{I_{s}} x_{k}^{0}\right| \leqslant \Sigma_{s}\left|\Delta_{I_{s}} x_{k}{ }^{+}\right|+\Sigma_{s}\left|\Delta_{I_{s}} x^{-}\right| \leqslant i+1+2 M_{0} b^{\nu} .
$$

We have proved that the interval functions $\Delta_{I} x^{-}, \Delta_{I^{x}} x_{k}^{+}, \Delta_{I} x_{k}^{0}$ are of bounded variation, namely, of equibounded variation in $R$. In addition, $\Delta_{T} x$ is obviously absolutely continuous in $R$. Note that, if $I_{t}=[0, t]$ denotes the interval $0 \leqslant \tau^{i} \leqslant t^{i}, i=1, \ldots, \nu$, in $R$, then,

$$
\left|x_{k}^{0}(t)\right|=\left|\Delta_{I_{i}} x_{k}^{0}\right| \leqslant i+1+2 M_{0} b^{p}, \quad t \in R,
$$

that is, the functions $x_{k}{ }^{0}(t)$ are equibounded in $R$, and so are the functions $x_{k}+(t)$.

Let us consider the countably many lattice points $\left\{t_{\rho}\right\}$, or $t=b \rho \in R$, $\rho=\left(\rho_{1}, \ldots, \rho_{v}\right)$, where $\rho_{1}, \ldots, \rho_{\nu}$ denote all rational numbers, $0 \leqslant \rho_{j} \leqslant 1$, $j=1, \ldots, \nu$, Let $\{I\}$ be the countable system of intervals $I \subset R$ whose vertices are points $t \in\left\{t_{\rho}\right\}$. We may order the points $t_{\rho}$ into a sequence. Since the functions $x_{k}{ }^{0}(t), x_{k}{ }^{+}(t), t \in R$, are uniformly bounded in $R$ (and, hence, at each $t=t_{\rho}$ ), we can successively select subsequences which are convergent at $t=t_{\rho}$ and then, by the diagonal process, we can select a unique subsequence $\left[k_{s}\right]$ of integers $k$ such that the limits $x_{k_{s}}^{0}(t) \rightarrow x^{0}(t), x_{k_{s}}^{+}(t) \rightarrow x^{+}(t)$ as $s \rightarrow \infty$ exist, for every $t \in\left\{t_{\rho}\right\}$. Here, $x^{-}(t)$ does not depend on $k$, and $\Delta_{T} x_{k}{ }^{0}=$ $\Delta_{I} x_{k}{ }^{+}+\Delta_{I} x^{-}$. Thus, as $k=k_{s} \rightarrow \infty, \Delta_{I} x_{k}{ }^{0}$ and $\Delta_{I} x_{k}{ }^{+}$have limits, say $\Delta_{I} x^{0}, \Delta_{I} x^{+}$, and $\Delta_{T} x^{0}=\Delta_{I} x^{+}+\Delta_{I} x^{-}$for every $I \in\{I\}$, and $\Delta_{I} x^{0}, \Delta_{I} x^{+}, \Delta_{I} x^{-}$ are additive interval functions in $\{I\}$. Moreover, $\Delta_{I} x^{-}$is absolutely continuous, $\Delta_{I} x^{+} \geqslant 0, \Delta_{I} x^{+}$is of bounded variation and, hence, $\Delta_{I} x^{0}$ is also of bounded variation in $\{I\}$.

By Ref. 8 , we know that $\Delta_{I} x^{0}$ is then the difference $\Delta$ of a function $x^{0}(t)$ defined everywhere in $R$, with a Lebesgue decomposition $x^{0}(t)=X(t)+S(t)$. Hence, $\Delta_{I} x^{0}=\Delta_{I} X+\Delta_{I} S$, where

$$
x^{0}\left(t_{i}^{\prime}, 0\right)=X\left(t_{i}^{\prime}, 0\right)=S\left(t_{i}^{\prime}, 0\right)=0, \quad\left(t_{i}^{\prime}, 0\right) \in \partial R, \quad i=1, \ldots, \nu,
$$

and where $\Delta_{I} X$ is absolutely continuous and $\Delta_{I} S$ is singular. Consequently, for every $t_{0} \in R$ and interval $I \in\{I\}$ with $t_{0} \in I$, we can form the quotients 
$\triangle_{I} S /$ meas $I$. We know that for $I$ a hypercube, $I \in\{I\}, t_{0} \in I$, and almost all $t_{0} \in R$, we have $\Delta_{i} S /$ meas $I \rightarrow 0$ as diam $I \rightarrow 0$. On the other hand, the same process applied to $\Delta_{I} X$ yields $\Delta_{I} X /$ meas $I \rightarrow z\left(t_{0}\right)$. Now, $z(t)$ is defined a.e. in $R$, is $L_{1}$-integrable in $R$, and

$$
\Delta_{I} X=\int_{I} z(t) d t, \quad I \in\{I\} .
$$

In addition, the decomposition $x_{k}^{0}=x_{k}^{+}+x^{-}$, with $\Delta_{I} x_{k}^{+} \geqslant 0, \Delta_{I} x^{-} \leqslant 0, x^{-}$ independent of $k$ and $\Delta_{1} x^{-}$absolutely continuous, implies $x^{0}=X+S=$ $X^{+}+x^{-}$, with $\Delta_{I} X^{+} \geqslant 0, \Delta_{I} x^{-} \leqslant 0$. By the uniqueness of the Lebesgue decomposition, we conclude that $\Delta_{1} X^{+}$has the Lebesgue decomposition $\Delta_{I} X^{+}=\Delta_{I}\left(X-x^{-}\right)+\Delta_{I} S$, where $\Delta_{I}\left(X-x^{-}\right)$is absolutely continuous and $\Delta_{Y} S$ is singular. Then $\Delta_{X} X^{+} \geqslant 0$ implies $\Delta_{I}\left(X-x^{-}\right) \geqslant 0, \Delta_{I} S \geqslant 0$, in particular $S(b)=\Delta_{R} S \geqslant 0$.

We can now apply Closure Theorem 2 with $s$ replaced by $n, n$ replaced by $n+1$, and $n-s$ replaced by 1 ; also, $x^{1}, \ldots, x^{s}$ is replaced by $x^{1}, \ldots, x^{n}$ and $x^{s+1}, \ldots, x^{n}$ is replaced by $x^{0}$. By Closure Theorem 2 with $x^{0}=X+S$, we conclude that there exists some measurable function $u(t)=\left(u^{1}, \ldots, u^{m}\right)$ $\in U(t, x(t)), x(t)=\left(x^{1}, \ldots, x^{n}\right)$, such that

$$
\begin{gathered}
(t, x(t)) \in A, \quad u(t) \in U(t, x(t)) \quad \text { a.e. in } G, \\
D^{\alpha} x^{i}(t)=f_{i \alpha}(t, x(t), u(t)) \quad \text { a.e. in } G, \quad \alpha \in\{\alpha\}_{i}, \quad i=1, \ldots, n, \\
D^{\alpha} X(t)=f_{0}(t, x(t), u(t)) \quad \text { a.e. in } G, \quad \alpha=\alpha_{0} .
\end{gathered}
$$

On the other hand, $x(t), u(t), t \in G$, is in $\Omega$ since $\Omega$ is complete and

$$
i \leqslant I[x, u]=X(b)=x^{0}(b)-S(b)=\lim _{s+\infty} x_{k_{s}}^{0}(b)-S(b)=i-S(b) \leqslant i .
$$

Thus, the equality sign holds everywhere in this relation, $S(b)=0, I[x, u]=i$, and, hence, $I[x, u]$ possesses an absolute minimum in $\Omega$.

\section{References}

1. Cesarr, L., Existence Theorems for Multidimensional Problems of Optimal Control, Differential Equations and Dynamical Systems, pp. 115-132, Academic Press, New York, 1967.

2. CESARI, L., Existence Theorems for Weak and Usual Optimal Solutions in Lagrange Problems with Unilateral Constraints, I and II, Transactions of the American Mathematical Society, Vol. 124, pp. 369-412 and 413-429, 1966. 
3. Cesari, L., Existence Theorems for Optimal Solutions in Pontryagin and Lagrange Problems, SIAM Journal on Control, Vol. 3, pp. 475-498, 1965.

4. Sobolev, S. L., Applications of Functional Analysis in Mathematical Physics, American Mathematical Society, Providence, Rhode Island, 1963.

5. Choquet, G., Convergences, Annales de l'Université de Grenoble, Vol. 23, pp. 55-112, 1947-48.

6. Kuratowski, C., Les Fonctions Semi-Continues dans l'Espace des Ensembles Fermés, Fundamenta Mathematicae, Vol. 18, pp. 148-166, 1932.

7. Michafl, E., Topologies on Spaces of Subsets, Transactions of the American Mathematical Society, Vol. 71, pp. 152-182, 1951.

8. Riesz, F., and NaGY, B. S., Functional Analysis, Ungar, New York, 1955.

9. Castaing, C., Quelques Problèmes de Mesurabilité Liés à la Théorie de la Commande, Comptes Rendus de l'Académie des Sciences, Vol. 262, Série A, pp. 409-411, 1966. 